Salud y Servicios sociales

\title{
Historia de las especialidades médicas en Nicaragua: génesis y
} perspectivas

\section{History of medical specialties in Nicaragua: genesis and perspectives}

\section{Fisher Chavarría, Edén Lenin}

Edén Lenin Fisher Chavarría

do1h56-mga@minsa.gob.ni

Hospital Escuela Antonio Lenin Fonseca Martínez,

Nicaragua

Revista Torreón Universitario

Universidad Nacional Autónoma de Nicaragua-Managua,

Nicaragua

ISSN: $2410-5708$

ISSN-e: $2313-7215$

Periodicidad: Cuatrimestral

vol. 11, núm. 30, 2022

tevis.torreon.faremc(eunan.edurini

Recepción: 09 Diciembre 2021

Aprobación: 22 Enero 2022

URL: http://portal.amelica.org/ameli

jatsRepo/387/3872816015/index.htm

DOI: https://doi.org/10.5377/rtu.v11i30.13426

El autor o los autores de los artículos, ensayos o investigaciones conceden a la Universidad Nacional Autónoma de Nicaragua, Managua (UNAN-Managua) los derechos de edición (copyright) del trabajo enviado, por consiguiente la Universidad cuenta con el derecho exclusivo para publicar el artículo durante el periodo completo de los derechos de autor.

\section{(i) $(9)$}

Esta obra está bajo una Licencia Creative Commons AtribuciónNoComercial-SinDerivar 4.0 Internacional.

\section{INTRODUCCIÓN}

La historia de las especialidades médicas en Nicaragua es un tema que los médicos, estudiantes de medicina y todos los profesionales y técnicos relacionados con la salud humana o biomedicina, deben conocer en sus aspectos fundamentales para entender como nuestro país ha venido avanzando, poco a poco, en profundizar

\section{NotAS DE AUTOR}


el conocimiento científico-técnico de los médicos para mejorar la calidad del sistema de salud nacional, en el sector público, de la seguridad social y en el sector privado.

Algunos aspectos históricos de la medicina nacional nos ayudan a entender mejor la relevancia y el impacto de la institucionalización de las especialidades médicas y quirúrgicas en el país. Los estudios de medicina en la región centroamericana comenzaron en Guatemala, en la Universidad San Carlos de Borromeo, donde el primer estudiante nicaragüense fue Francisco de Chavarría originario del puerto de El Realejo; pero no finalizó porque murió repentinamente durante una intensa discusión filosófica con su compañero de estudios Antonio Escobedo. Posteriormente, dos nicaragüenses más iniciaron y finalizaron sus estudios de medicina en Guatemala, ellos fueron: Timoteo Mayorga y Juan Bautista Muñoz (Montalván, 1960; Fisher, 2010a).

De la Huerta y Caso fundó la primera cátedra de medicina en Nicaragua, en 1798, cuya duración fue efímera (Pérez, Ulloa y Ramos, 2003) y se restableció en 1804 (Arellano, 2002). Debido a que el 15 de mayo de 1807 el Colegio Seminario de San Ramón fue elevado a Universidad Menor, por lo cual podía extender el título de Bachiller en Medicina, al día siguiente (16-5-1807) comenzó a impartir la cátedra de medicina, el doctor Francisco Quiñónez, graduado en la Universidad de San Carlos Borromeo de Guatemala (Arellano, 2002), y quien era, nada más y nada menos, que el único médico académico a lo largo y ancho de la Provincia de Nicaragua (Pérez y col., 2003).

El Protomedicato se formó el 20 de septiembre de 1848 y fue reglamentado en 1859, en León. Era una instancia de origen colonial que reorganizó los estudios de medicina (Montalván, 1960; Corea, 2000). A mediados de la década de 1880 la universidad asumió el modelo napoleónico o profesionalizante para satisfacer la necesidad de profesionales que el Estado y la oligarquía postcoloniales requerían, como sucedía en otros países latinoamericanos (Pérez y col., 2003).

En los últimos 25 años del siglo XIX algunos médicos nicaragüenses viajaron a Francia o Estados Unidos para estudiar medicina. Entre los más conocidos, miembros de las clases sociales más ricas del país, están: Luis Henry Debayle, graduado en París, y Juan José Martínez Moya, graduado en Nueva York. Estos médicos vinieron a aplicar las técnicas de antisepsia y a modernizar la medicina y la cirugía. Además, operaban diferentes regiones anatómicas del cuerpo humano, desde los ojos hasta la vesícula biliar (Fisher, 2011).

En los primeros ochenta años del siglo XX, los médicos nicaragüenses realizaron estudios de especialización en Estados Unidos, Francia, México, Brasil, Chile o Colombia. Optaban por una beca o asumían los gastos por su propia cuenta.

Mención especial merece el famoso trío o cuarteto de la Soborna, médicos nicaragüenses, que estudiaron en la famosa universidad de París y que regresaron a ejercer la medicina en la ciudad de León, a finales del año 1920; ellos fueron los siguientes: Humberto Tijerino, Alfonso Argüello, Alcides Delgadillo y Alberto Martínez (Berríos, 1994).

El primer médico nicaragüense, que viajó al extranjero, para realizar estudios de especialización en radiología fue el doctor Inocente Lacayo, quien regresó de Francia alrededor de 1925-1926 y ejerció la radiología en la capital Managua (Fisher, 2015).

Fue en 1955 que regresó al país el primer neurocirujano que tuvo Nicaragua, el doctor César Amador Kühl, graduado en Estados Unidos (Amador, 2012; Fisher, 2021a). Por otra parte, las cuatro primeras generaciones de oftalmólogos se especializaron en otros países (Fisher y Aragón, 2018).

Si las etapas históricas de la medicina en Nicaragua son siete: 1) mágica; 2) galénica; 3) mestiza; 4) pausteriana; 5) especializada; 6) social; y 7) moderna (Álvarez, 2013; Fisher, 2019a), entonces, la fundación y el impulso de las especialidades médico-quirúrgicas serían parte de la etapa moderna.

\section{Desarrollo}

En 1979 triunfó la Revolución Popular Sandinista. Debemos recordar que la historia de la medicina nacional indica que fue en 1982-1983, en plena Revolución Sandinista, que se inició el proceso de formación de las 
especialidades médicas y quirúrgicas, con certificación universitaria; antes de lo cual, los médicos tenían que viajar al extranjero, becados o con sus propios recursos económicos, para poder especializarse (Fisher, 2014).

Dicha iniciativa surgió del seno de la Dirección Nacional del FSLN, se transmitió a la Junta de Gobierno de Reconstrucción Nacional, luego al Consejo Nacional de la Educación Superior, según ha señalado el doctor en diseño experimental Manuel Enrique Pedroza Pacheco (Fisher, 2021a).

Posteriormente, el Ministerio de Salud concretó la decisión gubernamental en coordinación con la UNAN-León, universidad que inició la certificación universitaria de los títulos de los nuevos especialistas. Nicaragua necesitaba comenzar a formar y entrenar a sus propios médicos especialistas, en medio de nuestra propia realidad y con nuestros propios recursos materiales y humanos (Fisher, 2014). A ningún gobierno se le había ocurrido tomar una decisión de ese tipo. Cuando empezó tan gigantesca tarea, los enemigos de la Revolución Sandinista criticaban y se burlaban. La historia le ha dado la razón a los gobernantes sandinistas. Casi 40 años después los resultados están ahí, en miles de médicos especialistas atendiendo al pueblo en las distintas esferas de la medicina.

Sin olvidar que también se extendió títulos universitarios a los maestros de generaciones, con gran experiencia y conocimiento, así como a los médicos que se entrenaban de forma teórica y práctica en hospitales de Managua y León, ejerciendo la labor de médicos residentes, como era la costumbre hasta entonces, pero que no obtenían un título universitario al final de su residencia médica (Fisher, 2014).

Para que los programas de especialidades médicas y quirúrgicas se impulsaran, la Comisión Central conformada por el Minsa y la U.N.A.N., exigió que todos los planes y programas académicos estuvieran elaborados antes de 1982 (Guevara, 2003; Fisher, 2014).

Entre las 18 especialidades médicas y quirúrgicas que recibieron la acreditación universitaria, a partir del proceso transformador iniciado en la década de 1980, se pueden mencionar las siguientes: medicina interna, ginecología y obstetricia, cirugía, pediatría, ortopedia y traumatología, cirugía pediátrica, urología, psiquiatría, medicina física y rehabilitación, patología, otorrinolaringología, anestesiología, dermatología, cirugía plástica, oftalmología, radiología, cirugía maxilofacial y neurocirugía.

Además, se aumentó el número de estudiantes que ingresaban a estudiar medicina, en 100, 200 y 300\%; se fundó la UNAN-Managua, en 1984 y su Facultad de Medicina. A mediados de la década de 1970 el número de estudiantes de primer ingreso a la carrera de medicina, en León, estuvo entre 50 y 70 cada año académico.

La Facultad de Medicina, en Managua, surge como escuela en 1980, cuando 60 estudiantes de cuarto y quinto año de medicina de la UNAN, cuya sede estaba en la ciudad de León, se trasladan a recibir la formación médica en los hospitales médico-quirúrgicos de Managua. En 1981 se produce la apertura del primer año de la carrera de medicina, en Managua, en el Recinto Universitario Rubén Darío (Pérez y col., 2003).

Aumentar el número de estudiantes de medicina favoreció la formación de más médicos residentes en los programas de especialización médica o quirúrgica; pero con la seguridad de recibir un título universitario de especialista. La residencia es el período de estudio, entrenamiento y trabajo en que un médico y cirujano se especializa en una rama de la carrera de medicina, que se realiza generalmente en un ambiente hospitalario, que dura tres o cuatro años, tras lo cual una universidad entrega el título de especialista. La residencia es la mejor etapa de la vida y formación de un médico, dicen algunos; pero no todos los médicos piensan igual (Fisher, 2010b).

El residente o médico residente juega un doble papel: estudiante y profesional, trabajador. Como estudiante debe esforzarse disciplinadamente en aprender todos los conocimientos teóricos y prácticos de la especialidad que está estudiando. Y como trabajador debe cumplir un horario laboral diario de ocho horas y además hacer turnos nocturnos o durante los fines de semana. El trabajo es tanto (entre más se hace y se ve, más se aprende), que algunos afirman que el residente tiene hora de entrada, pero no tiene hora de salida. Por ese arduo ritmo de estudio y trabajo es recomendable hacer la residencia lo más joven posible (Fisher, 2010b). 
Durante la residencia se estimula erróneamente un sentido de competencia egoísta, lo cual es peligroso porque en toda competencia siempre alguien gana a costa de la derrota o pérdida de otro. De tal manera que, lo que debería existir durante la residencia es la cooperación (Fisher, 2010b).

A lo largo de 16 años de neoliberalismo ortodoxo se redujeron drásticamente las plazas o cupos de médicos residentes. El presidente Daniel Ortega entregó en 1990 un total de 270 plazas a la nueva presidenta, Violeta Barrios. En 2007, el nuevo presidente Daniel Ortega recibió de parte del presidente saliente, Enrique Bolaños, solo 87 plazas. Así, Nicaragua no pudo formar, por lo menos a 2500 médicos especialistas (Fisher, 2009).

A partir de 2007 se aumentaron las plazas para médicos residentes, llegando a ser 300 de nuevo ingreso. Asimismo, todos los residentes tuvieron derecho a una beca cuyo valor es en promedio de 500 dólares (Fisher, 2019b). Desde el año 2007 empezó a disminuir dicho número de nuevos residentes, en parte debido a las acciones de bloqueo económico sobre Venezuela, que afectaron algunos acuerdos comerciales con Nicaragua. Tal descenso se agravó con el golpe de Estado blando, que impulsaron las fuerzas de la extrema derecha, lo que causó 1400 millones de dólares en pérdidas al país y alrededor de 170 mil desempleados (Fisher, 2020).

Durante las casi cuatro décadas del programa nacional de especialización, muchos médicos, ya graduados como especialistas, han realizado, posteriormente, estudios de sub-especialización en otros países, principalmente latinoamericanos, como México, Guatemala, Brasil o Colombia (Fisher y Aragón, 2018).

En los últimos años, el Ministerio de Salud ha impulsado la especialización en emergenciología, cuidados intensivos o críticos, perinatología, gerencia de hospitales y radioterapia. La sub-especialización, o alta especialización, como le llaman en otros países, ha iniciado en el año 2021, en las áreas de cardiología, nefrología y neurología (Fisher, 2021b). Sería razonable, si existieran las condiciones materiales, que la subespecialización inicie en el último año de la especialidad, porque la demanda es alta, la población aumenta y la vida no es tan larga como parece (Fisher, 2011b).

Los hospitales escuela que han formado médicos especialistas desde la primera mitad de la década de 1980 hasta el día de hoy, son los siguientes: Oscar Danilo Rosales Argüello, en León. Por su parte, en Managua, han sido: Antonio Lenin Fonseca Martínez, Manolo Morales Peralta, Fernando Vélez Paiz, Manuel de Jesús Rivera, Bertha Calderón, Alejandro Dávila Bolaños, Alemán - Nicaragüense, Centro Nacional de Oftalmología, Psicosocial, Dermatológico, Aldo Chavarría. Posteriormente se han sumado los hospitales Bautista, Carlos Roberto Huembes, Centro Nacional de Radioterapia, Bolonia y Nuevo Monte España.

Las perspectivas son una ampliación del programa de especialización, así como la consolidación y ampliación de las sub-especialidades, sobre todo con la construcción de nuevos y modernos hospitales, para mejorar la atención en salud del pueblo nicaragüense, incluyente y gratuita, como solamente un gobierno revolucionario puede hacerlo, desde institucionalizó las especialidades médicas y quirúrgicas, en la década de 1980 (Fisher, 2021b).

\section{Conclusiones}

La historia de las especialidades médicas en Nicaragua se puede dividir en cuatro etapas. Primera, de 1920 a 1979 (especialización en el extranjero). Segunda, de 1979 a 1990 (fundación e institucionalización de la especialización nacional). Tercera, de 1990 a 2006 (estancamiento y retroceso de la especialización). Cuarta, de 2007 hasta nuestros días (revitalización de la especialización e inicio de la sub-especialización).

El país ha dado un salto cuantitativo y cualitativo en el número de médicos y cirujanos especialistas, articulando las políticas públicas a través del Ministerio de Salud y la Universidad Nacional Autónoma de Nicaragua, tanto de León como de Managua. El papel rector del Estado ha sido determinante. La Revolución Sandinista ha sido el principal fenómeno sociológico influyente en el complejo proceso de la especialización y sub-especialización de los médicos nicaragüenses, como un reflejo de la transformación revolucionaria del país a través de la educación en general, de la educación superior y de la educación médica en particular, para 
beneficiar a todos los ciudadanos con una mayor cobertura, más calidad y mayor especialización del personal médico.

\section{ReFERENCIAS}

Álvarez Montalván, E. (2013). Desarrollo de la medicina en Nicaragua. En: Médico de vocación y aficionado en política. Managua. La Prensa.

Amador Kühl, C.A. (2012). Memorias de mi vida. Fondo de Promoción Cultural Invercasa. Managua, Nicaragua.

Arellano, J. E. (2002). Interpretación de la leonesidad. En: Boletín nicaragüense de bibliografía y documentación. Rubén Darío y la leonesidad. Banco Central de Nicaragua. Imprimatur. Managua. Ene-Mar: 114

Berríos Valladares, G. (1994). 90 años de cirugía en León. Bolsa Médica. No. 6; Feb.: 4-6

Corea Fonseca, E. (2000). Historia de la medicina en Nicaragua. La Prensa. Managua.

Fisher, L. (2009). Sobre las especialidades médicas: respuesta a un ex-Decano. En: La revolución antineoliberal. Editronic. Managua, Nicaragua.

Fisher, L. (2010a). Universidad de Nicaragua: reseña histórica. En: Historia de la radiología en Nicaragua: la senda de la luz invisible. Universitaria. Managua.

Fisher, L. (2010b). La residencia. En: Historia de la radiología en Nicaragua: la senda de la luz invisible. Universitaria. Managua.

Fisher, L. (2011a). Los rayos X en Nicaragua. En: Historia de la radiología en Nicaragua. 2da. ed. Universitaria. Managua.

Fisher, L. (2011b). Especialización dentro de la radiología. En: Historia de la radiología en Nicaragua. 2da. ed. Universitaria. Managua.

Fisher, L. (2014). Revolución Sandinista y especialidades médico-quirúrgicas. En: Escritos revolucionarios del siglo XXI. Universitaria. Managua, Nicaragua.

Fisher, L. (2015). La radiología nicaragüense: 113 años de historia. Universitaria. Managua.

Fisher, L. Aragón Padilla, J.P. (2018). La historia de la oftalmología y la optometría en Nicaragua. Universitaria Tutecotzimí. Managua.

Fisher, L. (2019b). Médicos residentes y políticas públicas en Nicaragua. Colegio Nicaragüense de Radiología. https:// colegionicaraguenseradiologia.blogspot.com/: 3-3-19

Fisher, L. (2019a). Etapas de la medicina nicaragüense. Colegio Nicaragüense de Radiología. https:// colegionicaraguenseradiologia.blogspot.com/: 27-8-19

Fisher, L. (2020). Golpe de Estado blando, Nicaragua 2018: otra victoria del FSLN. Universitaria. Managua.

Fisher, L. (2021a). Historia de la neurocirugía en Nicaragua. Universitaria. Managua.

Fisher, L. (2021b). Subespecialidades médicas en Nicaragua. Colegio Nicaragüense de Radiología. https:// colegionicaraguenseradiologia.blogspot.com/: 2-4-21

Guevara Guerrero, U. (2003). Historia de la patología en Nicaragua. Universitaria. León.

Montalván, L. H. (1960). Breves apuntes sobre deontología médica e historia universal de la medicina. Hospicio. Universidad Nacional Autónoma. León, Nicaragua.

Pérez Orozco, G., Ulloa González, A., Ramos Vega, D. (2003). Recordando con el corazón...construimos el futuro: 23 años de historia de la Facultad de Medicina, UNAN-Managua. Imprimatur. Managua. 